\title{
Paraspinal Pyomyositis Associated with Radiculopathy
}

\author{
Takeshi Kondo ${ }^{1}$, Toshihiko Takada ${ }^{1,2}$, Kazuhiko Terada ${ }^{2}$ and Masatomi Ikusaka ${ }^{1}$
}

\begin{abstract}
A 57-year-old woman presented with a fever and left-sided hip and back pain. The hip pain was explained by L3 radiculopathy because the pain was distributed on the L3 dermatome accompanied by allodynia. On the other hand, the back pain was located on the left side $1 \mathrm{~cm}$ from the spinal process of the L3-4 vertebrae. Enhanced computed tomography of the abdomen revealed an abscess in the left paravertebral muscle, and pyomyositis was diagnosed. Following percutaneous drainage via ultrasound, both the hip and back pain immediately improved. Paraspinal pyomyositis as well as lesions inside the spinal canal should therefore be considered in the differential diagnosis of patients presenting with fever, back pain, and radiculopathy.
\end{abstract}

Key words: pyomyositis, radiculopathy, Streptococcus pneumoniae, back pain, hip pain

(Intern Med 52: 1417-1418, 2013)

(DOI: 10.2169/internalmedicine.52.0456)

\section{Introduction}

Pyomyositis is a purulent infection of the skeletal muscle that generally presents with fever and muscle pain. We herein report the case of a patient with left-sided hip and back pain who was diagnosed with pyomyositis associated with radiculopathy. To our knowledge, this is the first case report of pyomyositis with radiculopathy.

\section{Case Report}

In April 2011, a 57-year-old woman was admitted to the Orthopaedics Department with left-sided hip and back pain. In 2005, she had undergone total left hip replacement for osteoarthritis. The pain had begun the day before admission and became severe enough to disturb the patient's sleep. After admission, the pain was controlled with diclofenac. Hip and lumbar radiographs, plain computed tomography (CT) of the pelvis, and magnetic resonance imaging (MRI) of the lumbar spine did not reveal the cause of the pain. Two days after admission, the patient developed a fever and was referred to our department.

On examination, the pain was not exacerbated by moving the hip joint, and Patrick's test was negative. The pain was felt in the L3 dermatome accompanied by allodynia; therefore, we concluded that the cause of the hip pain was L3 radiculopathy. The patient also had back pain located approximately $1 \mathrm{~cm}$ to the left of the spinal processes of the L3-4 vertebrae that was exacerbated by movement and associated with severe tenderness.

In patients with back pain and radiculopathy, lesions inside the spinal canal, such as epidural abscesses, are generally considered; however, lesions in the canal at L3-4 would be expected to cause L4-5 radiculopathy. Therefore, the presence of lesions inside the spinal canal does not anatomically explain the development of radiculopathy at the same level, although lesions located outside of the canal could cause this phenomenon. We therefore considered the presence of lesions located outside of the canal, especially in the paravertebral muscles. MRI of the lumbar spine (Fig. 1) was initially reported as normal; however, slight swelling of the paravertebral muscles was identified retrospectively. Enhanced CT of the abdomen revealed an abscess in the left paravertebral muscles at the L3-4 level (Fig. 2), and pyomyositis was diagnosed. MRI of the left thigh ruled out local abscesses.

Following percutaneous drainage performed under ultrasound guidance, the patient's hip and back pain immediately improved. Blood cultures and the pus obtained during drainage grew Streptococcus pneumoniae. The patient responded to treatment with ceftriaxone ( $2 \mathrm{~g}$ twice daily) for three weeks. As of June 2012, no relapse had occurreed.

${ }^{1}$ Department of General Medicine, Chiba University Hospital, Japan and ${ }^{2}$ Department of General Medicine, Kimitsu Chuo Hospital, Japan Received for publication February 24, 2013; Accepted for publication February 27, 2013

Correspondence to Dr. Takeshi Kondo, reds_liverpool_2005@yahoo.co.jp 


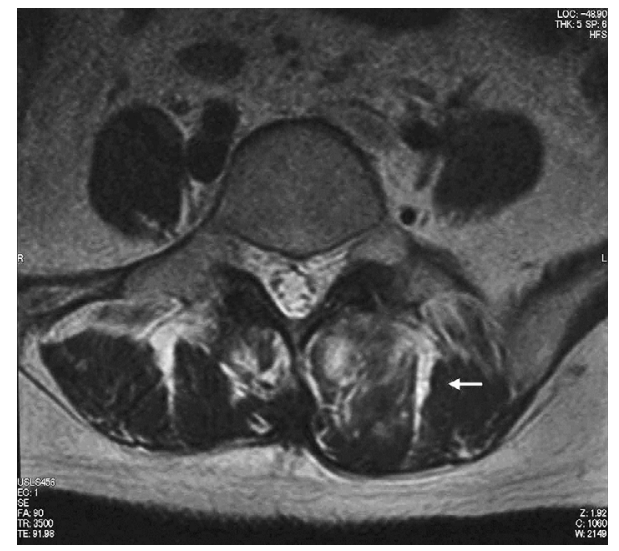

Figure 1. Plain MRI of the lumbar spine obtained on the day of admission showing swelling of the paravertebral muscles at L3-4 (arrow).

\section{Discussion}

Pyomyositis is a purulent infection of the skeletal muscles that usually manifests as single or multiple abscesses. It is classically a tropical disease; however, it has also been increasingly recognized in countries with temperate climates due to the increasing number of immunocompromised hosts, such as patients with human immunodeficiency virus infection or diabetes mellitus and those receiving immunosuppressive therapy. The disease can affect any age group. The presenting symptoms include fever and muscle pain. Common sites of infection are the lower extremity muscles, paraspinal muscles, iliopsoas muscle and upper extremity muscles (1). Staphylococcus aureus is the most common causative organism, while pyomyositis caused by Streptococcus pneumoniae is very rare (1). When compared with diseases caused by other microorganisms, the characteristics of pneumococcal pyomyositis include an older age of occurrence, female predominance, a severe clinical course, involvement of the psoas muscle, and predominance in temperate regions (2). Our patient had no risk factors for pneumococcal infection, such as splenectomy. Transthoracic echocardiography revealed no signs of infective endocarditis. MRI generally exhibits a higher sensitivity than $\mathrm{CT}$ with respect to making a diagnosis of pyomyositis (3). In our case, MRI of the lumbar spine was initially reported as normal

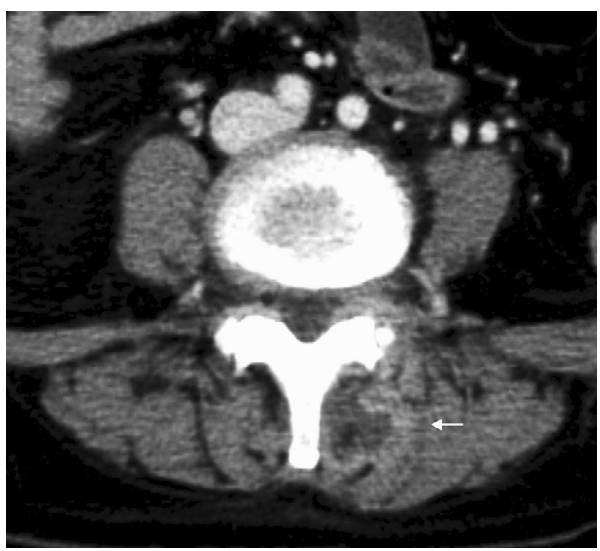

Figure 2. Enhanced CT of the abdomen obtained five days after admission showing a paravertebral muscle abscess at L3-4 (arrow).

because the disease was at a very early stage and changes were minimal. Such findings can easily be missed unless lesions of the paraspinal muscles are considered. For patients without other sequelae of bacteremia, treatment with drainage and three to four weeks of intravenous antibiotics is usually sufficient (1).

To our knowledge, paraspinal pyomyositis associated with radiculopathy has not been previously reported. Paraspinal pyomyositis as well as lesions inside the spinal canal must be considered in patients with fever, back pain and radiculopathy. Whether the lesions exist inside or outside of the canal can be determined from the level of back pain and radiculopathy.

The authors state that they have no Conflict of Interest (COI).

\section{References}

1. Crum NF. Bacterial pyomyositis in the United States. Am J Med 117: 420-428, 2004.

2. Collazos J, Fernández A, Martínez E, Mayo J, de la Viuda JM. Pneumococcal pyomyositis. Case report, review of the literature, and comparison with classic pyomyositis caused by other bacteria. Arch Intern Med 156: 1470-1474, 1996.

3. Theodorou SJ, Theodorou DJ, Resnick D. MR imaging findings of pyogenic bacterial myositis (pyomyositis) in patients with local muscle trauma: illustrative cases. Emerg Radiol 14: 89-96, 2007.

(C) 2013 The Japanese Society of Internal Medicine http://www.naika.or.jp/imonline/index.html 